\title{
Complete genome sequence of community-associated methicillin-resistant Staphylococcus aureus (strain USA400-0051), a prototype of the USA400 clone
}

\author{
Marina Farrel Côrtes ${ }^{1}$, Maiana OC Costa ${ }^{2}$, Nicholas CB Lima ${ }^{2}$, Rangel C Souza ${ }^{2}$, Luiz GP Almeida ${ }^{2}$, \\ Luciane Prioli Ciapina Guedes², Ana TR Vasconcelos², Marisa F Nicolás², Agnes MS Figueiredo ${ }^{1 /+}$ \\ ${ }^{1}$ Universidade Federal do Rio de Janeiro, Instituto de Microbiologia Paulo de Góes, \\ Laboratório de Biologia Molecular de Bactérias, Rio de Janeiro, RJ, Brasil \\ ${ }^{2}$ Laboratório Nacional de Computação Científica, Petrópolis, RJ, Brasil
}

Staphylococcus aureus subsp. aureus, commonly referred as S. aureus, is an important bacterial pathogen frequently involved in hospital- and community-acquired infections in humans, ranging from skin infections to more severe diseases such as pneumonia, bacteraemia, endocarditis, osteomyelitis, and disseminated infections. Here, we report the complete closed genome sequence of a community-acquired methicillin-resistant $S$. aureus strain, USA400-0051, which is a prototype of the USA400 clone.

Key words: methicillin-resistant Staphylococcus aureus - whole genome - USA400 - ST1-SCCmecIV

Methicillin-resistant Staphylococcus aureus (MRSA) isolates from the ST1-SCCmecIV lineage are regularly associated in healthy community-dwelling individuals. These isolates were first reported in Australia and named as Western Australia-1 clone (Udo et al. 1993). Later, ST1-SCC$m e c I V$ MRSA carrying lukSF-PV genes encoding the Panton-Valentine leucocidin (PVL) were detected in the USA and caused severe infections among American Indian children. The complete closed genome sequence of a representative of this MRSA, MW2, is deposited in GenBank (Baba et al. 2002). Years later, ST1-SCCmecIV MRSA $\left(\mathrm{PVL}^{+}\right)$re-emerged in the USA and Canada as an important cause of skin-soft tissues infections and was renamed as USA400 (McDougal et al. 2003, Golding et al. 2011). Here, we report the complete closed genome sequence of strain USA400-0051, a prototype of the USA400 clone, isolated during CA-MRSA outbreaks (2003-2006) of skin and soft tissue infections in the USA (Tenover et al. 2006).

Genomic DNA was obtained (Sambrook et al. 1989), and its concentration and purity were assessed using a Qubit ${ }^{\circledR} 2.0$ fluorometer (Invitrogen, Carlsbad, CA, USA). The library was prepared with $100 \mathrm{ng}$ DNA. Adapter ligation, size selection, nick repair, and amplification were performed using the Ion Xpress Fragment Library Kit (Ion Torrent; Thermo Fisher Scientific, Waltham, MA, USA). The Ion Sequencing Kit v2.0 was used for all sequencing reactions following the manufacturer's recommendations. Torrent Suite 1.5 was used for the analysis, and sequenc-

\footnotetext{
doi: 10.1590/0074-02760170128

Financial support: FAPERJ (\# E-26/010.001764/2014, E-26/201.147/2014), CNPq (\# 303067/2015-2)

+Corresponding author: agnes@micro.ufrj.br

Received 1 April 2017

Accepted 30 June 2017
}

ing was performed using 316 chips. The assembly, based on 741,383 reads, was carried out using Newbler v 2.6 (Roche Diagnostics, Basel, Switzerland) and Celera genome assembly v 6.1 (JCV Institute; Myers et al. 2000). Gaps within the scaffolds were resolved using genome sequencing performed using the 454 GS FLX titanium (3-kb paired-end library) approach (Roche). The genome was annotated using Sabia (Available from: www.sabia. lncc.br/; last access in August 2016) pipeline.

The genome of USA400-0051 consists of one circular chromosome with 2,832,530 base pairs (bp) and a GC content of $30.58 \%$. A total of 2773 protein-coding sequences were annotated (2299: known functions, 392: unknown categories, and 81: pseudogenes). The genome harbours 16 rRNA genes (five copies of $16 \mathrm{~S}$ rRNA, five of 23S rRNA, and six of 5S rRNA) and 40 tRNA genes, identified using RNAmmer and tRNAscan-SE (Schattner et al. 2005), respectively. The genome also contains a 2064-bp plasmid with a GC content of $28.75 \%$.

The USA400-0051 strain was typed as spa t128 and SCCmecIVa (ccrA2 and ccrB2). This strain harbours most of the $S$. aureus adhesion- and biofilm-associated genes: ebh, clfA, clfB, cna, ebp, map, efb, fnbAB, icaAB$C D R, s d r C D E$, and $s p a$, in addition to atl. The genome of USA400-0051 also contains an arsenal of enterotoxin and enterotoxin-like genes such as sea, sec, seh, and sek and selq selk, and sell, respectively, in addition to other toxin-associated genes including sak, hla, hlb, hld, hlgA, $h \lg B, h \operatorname{lgC}$, snc, eta, lukSF-PV, lukDE, and lukXY (encoding staphylokinase, $\alpha-, \beta$ - and $\delta$-hemolysins, staphylococcal bi-component $\gamma$-hemolysin A, B, and C, staphylococcal complement inhibitory precursor, exfoliative toxin $\mathrm{A}$, and the leucocidins Panton-Valentine-PVL, LukDE and LukXY; respectively). These virulence genes are mostly present in mobile genetic elements such as bacteriophages [(i) PhiSa2mw carrying the PVL genes (lukSF) and (ii) PhiSa3mw containing sea, selk, and selq] and genomic islands [(GI) (i) SAPImw2 carrying 


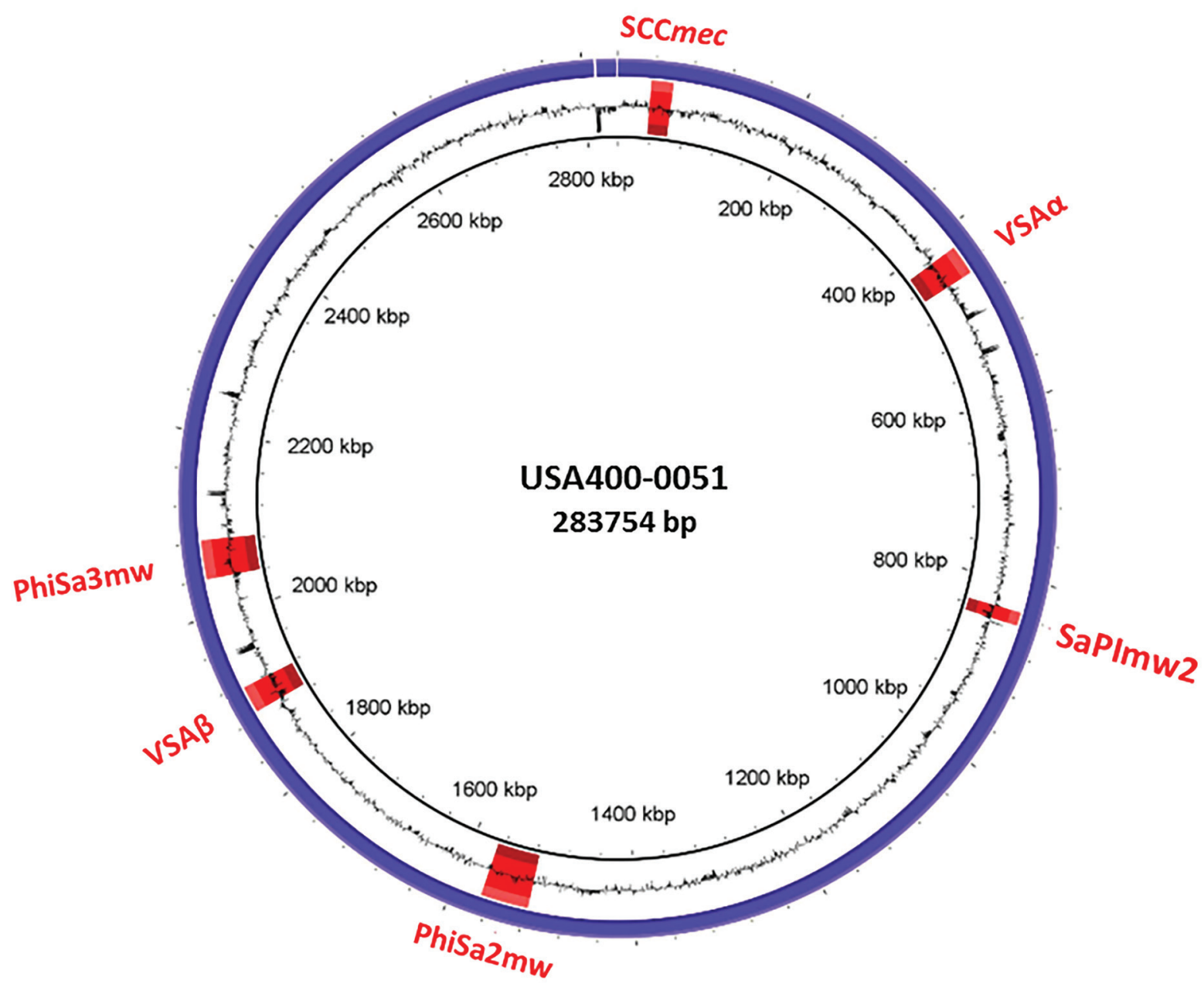

Fig. 1: genomic atlas of strain USA400-0051 created using BRIG. The blue circle represents the genomic sequence and the black circle indicates the GC content. In red are the positions of the genomic island SCCmec, vSA $\alpha$, SaPImw2, and vSA $\beta$ and bacteriophages PhiSa2mw and PhiSa3mw.

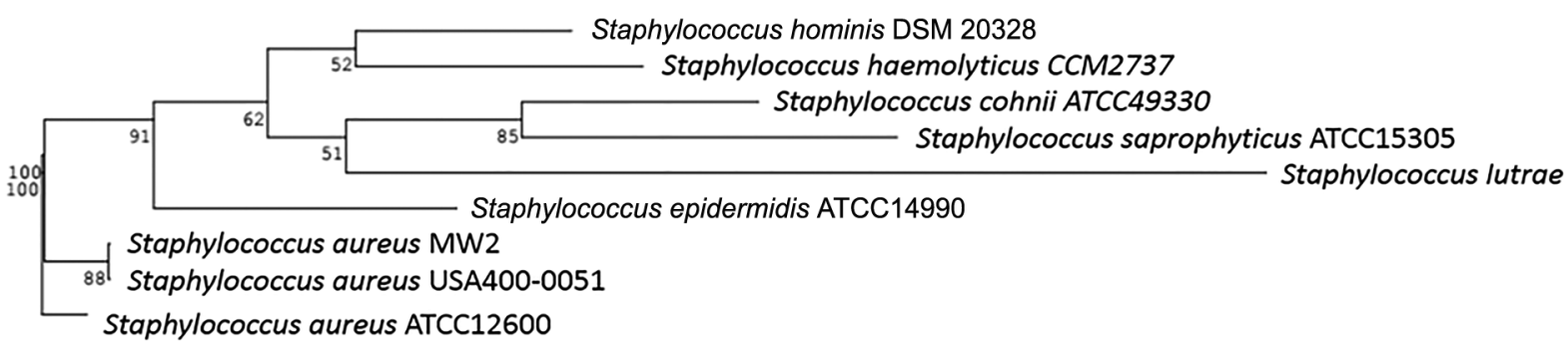

Scale: $\longmapsto$

Fig. 2: phylogenetic tree. ATCC 12600 (NCBI access: L36472), ATCC 15305 (NCBI access: AP008934), ATCC 14990 (NCBI access: D83363), DSM 20328 (NCBI access: X66101), CCM2737 (NCBI access: X66100), ATCC 49330 (NCBI access: AB009936). Staphylococcus lutrae sequence (NCBI access: X84731) was used as the outgroup. 

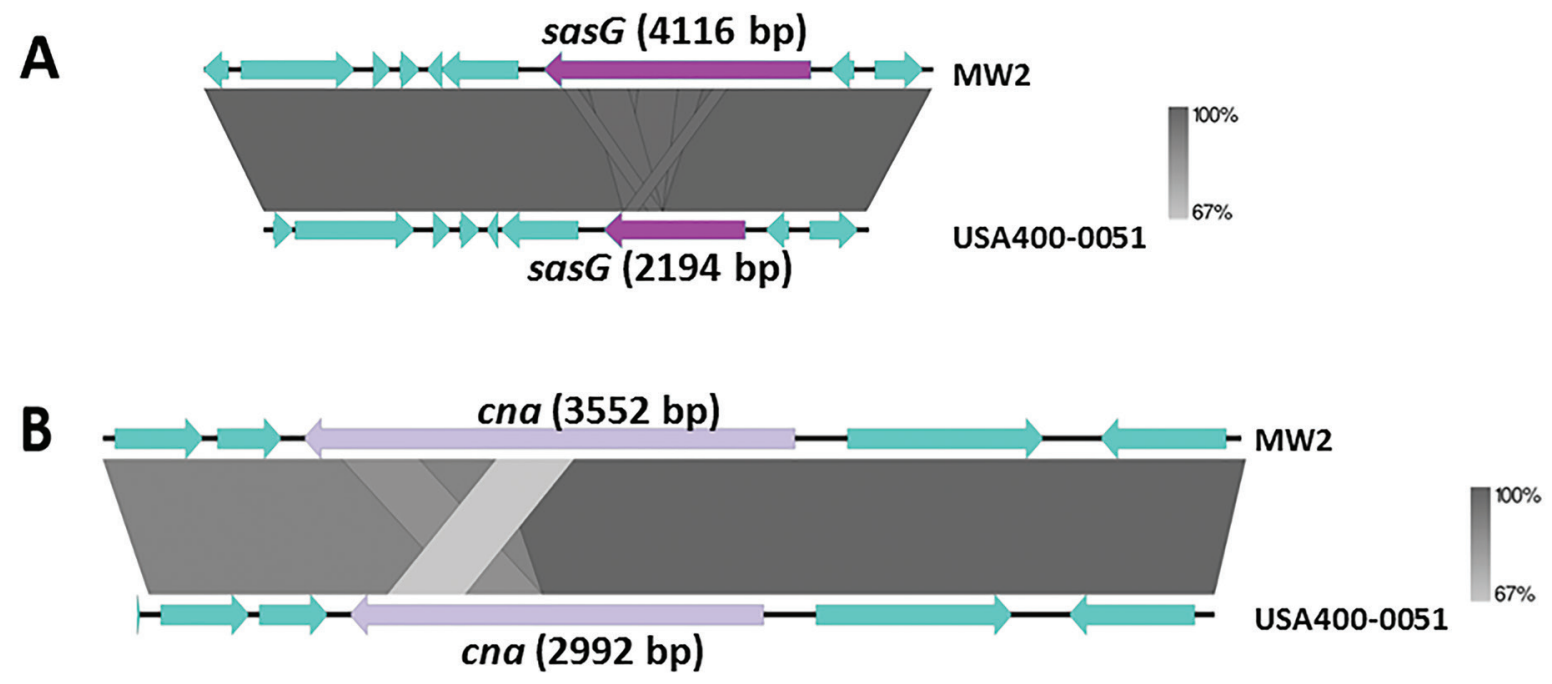

Fig. 3: alignment of the context region comprising the genes $\operatorname{sas} G$ (A) and $\operatorname{csn}(\mathrm{B})$ in strain USA400-0051 and closely related MW2 strain.

ear, sec, and sell; (ii) a type II vSA $\alpha$ carrying the gene clusters encoding staphylococcal superantigens set16, set17, set18, set19, set20, set $21, \operatorname{set} 22, \operatorname{set} 23, \operatorname{set} 24, \operatorname{set} 25$, and set 26 and lipoproteins lpl10, lpl11, lpl12, lpl13, and lpl14; and (iii) a type II vSA $\beta$ with an incomplete operon of the serine proteases splABCF and the lantibiotics epidermin locus $b s a A 1, b s a A 2, b s a B$ bsaD, bsaP, bsaF, $b s a E$, and $b s a G]$ (Fig. 1). Analyses using ResFinder 2.1 (www.cge.cbs.dtu.dk/services/ResFinder; last access in January 2016) showed that USA400-0051 is only resistant to $\beta$-lactam drugs and contains the genes blaZ and $m e c A$; this was confirmed by an antimicrobial disc susceptibility test. A tree was built based on the $16 \mathrm{~S}$ rRNA genes with RDP Tree Builder (www.rdp.cme. msu.edu/treebuilder/treeing.spr; last access in June 2016), using Weighbor with an alphabet size of four and length of 1000 . The bootstrapping process was repeated 100 times to generate a strict consensus tree (Cole et al. 2007). Fig. 2 shows the phylogenetic position of USA400-0051 in relation to other Staphylococcus sp. genomes. The phylogenetic tree showed that USA4000051 clustered with MW2 (the reference strain). The MW2 and USA400-0051 genomes showed few differences. Partial sas $G$ and cna genes were present in the USA400-0051 genome, which lacked a 560-bp fragment for cna and a 1922-bp fragment for sas G (Fig. 3A-B). In addition, MW2 harbours one additional copy of $16 \mathrm{~S}$ and $23 \mathrm{~S}$ rRNAs compared to USA400-0051.

The complete genome sequence of the USA400-0051 strain was deposited in the GenBank (WGS database) under NCBI accession number (chr: CP019574; plm: CP019575).

\section{AUTHORS' CONTRIBUTION}

MFC carried out the experiments to obtain total DNA for the sequencing reactions and was responsible for manual annotation, specialised annotations, and comparative genomics between the MW2 and USA300-0051 genomes. In addition, MFC wrote the draft version of the manuscript. LPCG carried out the sequencing reactions; RCS, LGPA and ATRV performed genome assembly and closed gapped regions in draft assemblies; MOCC and MFN carried out automatic and some manual annotations; AMSF was responsible for the study design, concluding analyses, and for approving the final version of the manuscript. All authors revised the final version of the manuscript.

\section{REFERENCES}

Baba T, Takeuchi F, Kuroda M, Yuzawa H, Aoki K, Oguchi A, et al. Genome and virulence determinants of high virulence community-acquired MRSA. Lancet. 2002; 359(9320): 1819-27.

Cole JR, Chai B, Farris RJ, Wang Q, Kulam-Syed-Mohideen AS, McGarrell DM, et al. The ribosomal database project (RDP-II): introducing myRDP space and quality controlled public data. Nucleic Acids Res. 2007; 35: D169-72.

Golding GR, Levett PN, McDonald RR, Irvine J, Quinn B, Nsungu $\mathrm{M}$, et al. High rates of Staphylococcus aureus USA400 infection, Northern Canada. Emerg Infect Dis. 2011; 17(4): 722-5.

McDougal LK, Steward CD, Killgore GE, Chaitram JM, McAllister SK, Tenover FC. Pulsed-field gel electrophoresis typing of oxacillin resistant Staphylococcus aureus isolates from the United States: establishing a national database. J Clin Microbiol. 2003; 41(11): 5113-20.

Myers EW, Sutton GG, Delcher AL, Dew IM, Fasulo DP, Flanigan $\mathrm{MJ}$, et al. A whole-genome assembly of drosophila. Science. 2000; 287(5461): 2196-204.

Sambrook J, Fritch EF, Maniatis T. Commonly used techniques in molecular cloning. Molecular cloning: a laboratory manual. Vol. III. New York: Cold Spring Harbor Laboratory Press; 1989. E3-4.

Schattner P, Brooks AN, Lowe TM. The tRNAscan-SE, snoscan and snoGPS web servers for the detection of tRNAs and snoRNAs. Nucleic Acids Res. 2005; 33: W686-9.

Tenover FC, McDougal LK, Goering RV, Killgore G, Projan SJ, Patel $\mathrm{JB}$, et al. Characterization of a strain of community-associated methicillin-resistant Staphylococcus aureus widely disseminated in the United States. J Clin Microbiol. 2006; 44(1): 108-18.

Udo EE, Pearman JW, Grubb WB. Genetic analysis of community isolates methicillin-resistant $S$. aureus in Western Australia. J Hosp Infect. 1993; 25(2): 97-108. 